Absorption bei der Darstellung des thierischen Gummis. Hier wird das letztere häufig von dem Eiweisscoagulum eingeschlossen, so dass es vollständig verschwunden zu sein scheint. Die Flüssigkeit kann dann gar keine Reaction auf Gummi mehr geben, während in dem ursprünglichen Auszuge eine beträchtliche zu constatiren war.

Die Vergeblichkeit der Bemïhungen, aus den Fällungen mit schweren Metallsalzen auf die Grösse des Peptons und Eiweissmolecüls zu schliessen, hat sicher ihren Grund darin, dass es sich auch hier nicht um chemische Verbindungen, sondern um mechanische Absorption handelt.

(Physiologisches Laboratorium in Bonn.)

Ueber die Bestimmung des Harnstoffs im menschlichen Harne nach der Methode von Knop-Hüfner.

Kritische Untersuchung

Von

E. Pflüger and Friedrich Schenck.

Nachdem die Brom-Methode von Knop-Hüfner in letzter Zeit sowohl von F. Falck') wie von Arnold ${ }^{2}$ ), welche sie kritisch experimentell prïften, als zu wissenschaftlichen Analysen ungeeignet bezeichnet worden war, versuchte neuerdings der cand. med. Carl Jacoby ${ }^{3}$ ) "aufgefordert von Herrn Professor Hüfner" nach dessen Methode und in dessen Laboratorium verschiedene Versuchsreihen zu deren Rechtfertigung durchzuführen. 21, 299.

1) Dies Archiv 26, 391. - Fresenius, Zeitschr. f. analyt. Chem.

2) Arch. d. Pharm. 1882, p. 356, - Fresenius a. a. 0. 21, 606.

3) Fresenius, Zeitsehr. f. analyt. Chem. 24, p. 310. 
Jacoby gelangt zu dem Ergebniss, dass die Brom-Methode von Knop-Hüfner zu genaten wissenschaftlichen Untersuchungen allerdings geeignet sei, was von der Li ebig-Pflüger'schen Quecksilber-Methode nicht gelte. Letztere liefere weder richtige Werthe für den Harnstoff, noch für den Gesammtstickstoff ${ }^{1}$ ).

Was die Liebig-Pflüger'sche Methode leistet und wie gross im Allgemeinen and Speciellen der Beobachtungsfehler derselben ist, haben neuerdings Pflïger und Bohland in einer ausfübrlichen Untersuchung gezeigt und zugleich dargethan, dass diese Methode nicht eine Titration des Harnstoffs, sondern des gesammten im Harn enthaltenen Stickstoffs repräsentirt, wodurch ihr Werth erhöht wird. Damit ist die eine Hälfte obiger Behauptung Jacoby's widerlegt.

Es fragt sich nun, wie es mit dem Beobachtungsfehler der Methode von Knop-Hüfner steht.

Hüfner hat sich selbst überzengt, dass die Bromlauge den gesammten Stickstoff aus reinen Harnstofflösungen niemals liefert, weshalb er das Deficit von 4,2\% hinzu addiren muss.

Wenn, wie auch wir gefunden haben, diese Correctur bei Harnstofflösungen ohne Bedenken ist, so stellt sich das Verhältniss anders, sobald es sich um den Harn selbst handelt.

Denn es ist sogar in Hüfner's Laboratorium wieder von Jacoby der Nachweis geliefert worden, dass die Mengen gasförmigen Stickstoffs, welche durch Bromlauge aus einer Harnstofflösung in Freiheit gesetzt werden, wesentlich abhängen von der Gegenwart gewisser anderer Stoffe.

Jacoby sagt ${ }^{2}$ :

„Ans vorstehenden Versuchen ergibt sich in der That das unzweifelhafte Resultat, dass Gegenwart von Traubenzucker die Entbindung des Stickstoffgases befördert; dass auch, je grösser der Zuckergehalt, um so grösser im Allgemeinen jener förderliche Einfluss ist; dass indessen selbst ein Zuckergehalt von $6 \%$ das Freiwerden der gesammten von der Theorie geforderten Stickgasmenge noch nicht zu bewirken vermag."

Jac oby sagt ferner $^{3}$ ):

1) Jacoby, a. a. 0. p. 328 .

2) Jacoby, a. a. 0. p. 317.

3) Ja coby, a. a. O. p. 318. 
Ueber die Bestimmung des Harnstoffs im menschlichen Harne etc. 327

„Dureh die Gegenwart des Acetessigäthers wird also bei blossen Harnstofflösungen das gewöhnliche Deficit nahezu vollkommen beseitigt."

Demgemäss setzt die Anwendung der Hüfner'schen Correctur voraus, dass im normalen Harne keine Substanz vorkommt, welche ähnlich wie Zucker oder Acetessigäther bei Anwendung von Bromlauge wirkt. Dieser Nachweis ist nicht erbracht und die Kenntniss der chemischen Zusammensetzung des Harnes lange nicht abgeschlossen, sodass uns noch manche Ueberraschung bevorsteht.

Da aber ferner die Bromlauge nicht bloss aus Harnstoff, sondern, wie Hüfner, Schleich, Foster, Magnier, Esbach, Falck u. Andere nachwiesen, auch aus den Ammoniaksalzen, der Harnsäure, dem Kreatinin und Kreatin $\mathbf{u}$. s. w. einen mehr oder weniger grossen Theil des in ihnen enthaltenen Stickstoffs frei macht, so scheint die Methode von Knop-Hüfner den Werth für den Harnstoff zu gross, für den Gesammtstickstoff zu klein ergeben zu müssen.

Bei dem heutigen Zustande der Kenntnisse sind Schätzungen über die Grösse des Beobachtungsfehlers, wie sie versucht worden sind, trügerisch.

Hier muss positive Arbeit entscheiden. Wir wollen demnach in diesem Aufsatze genau feststellen, wie gross der Beobachtungsfehler ist, wenn man den nach der Knop-Hüfner'schen Methode gefundenen Stickstoff mit dem gesammten im Harn enthaltenen vergleicht.

Eine spätere Untersuchung soll den im Harne wirklich entbaltenen Harnstoff vergleichen mit dem nach Knop-Hüfner ermittelten.

Bei der grossen Beliebtheit, deren sich die Hüfner'sche Methode erfreut, wird es zunächst nöthig sein, wenn wir dem Leser die Fehler vorführen, welche begangen werden, wenn man so verfährt, wie es von jeher von allen Lehrbiichern der physiologischen Chemie vorgeschrieben worden ist.

Das Recept für die Bereitung der Knop'schen Lauge lautet $\operatorname{nach} \mathrm{Knop}^{1}$ ):

„Die Lösung von unterbromigsaurem Natron erhält man, in-

1) Berichte der Königl. sächs. Gesellsch. d. Wissensch. math.-phys. Classe 1870 , p. 12. 
dem man $100 \mathrm{gr}$ Natronhydrat in $250 \mathrm{cem}$ Wasser löst, vollkommen kalt werden lässt und der Lauge $25 \mathrm{ccm}$ Brom beimischt."

Von dieser Lauge reichen $50 \mathrm{ccm}$, die man mit $200 \mathrm{ccm}$ Wasser verdünnt hat, hin, um 130 bis $150 \mathrm{ccm}$ Stickstoffgas aus einer Salmiaklösung zn entwickeln.

Da es sich nun bei dem Hüfner'schen Verfahren um die Entbindung von circa $18 \mathrm{ccm}$ Stickstoff (aus $5 \mathrm{ccm}$ einer 1\%igen U-lösung) handelt, so bielt man sich für berechtigt, die Knop'sche Lauge auf das 5 fache zu verdiunnen, und die gewöhnliche Vorschrift lautete:

100 gr Natronhydrat, 250 cem Wasser, Abwartung der Erkaltung, Zusatz von $25 \mathrm{ccm}$ Brom, Verdünnung mit Wasser auf $1200 \mathrm{ccm}$.

So findet sich die Vorschrift in sämmtlichen neuesten Lehrbuichern.

Die nach diesen Vorsehriften ausgeführten Analysen sollen nun übersichtlich tabellarisch geordnet vorgefuihrt werden:

Siehe Tabelle I p. 329.

Das ausserordentlich grosse Deficit an Stickstoff setzte uns in Erstaunen. Es ist allerdings die von Hüfner verlangte Correctur nicht angebracht. Wenn man aber auch an dem grossen Werthe noch die dem Beobachtungsfehler von $4,2 \%$ entsprechende Correctur anbringt, so bleibt eine colossale Differenz uibrig.

Obwohl wir nun ganz nach Hïfner's Vorschriften mit dessen Apparat arbeiteten, hielten wir es doch für nothwendig, den Correctionscoefficienten für unsere Langen und Versuchsbedingungen durch besondere Versuche festzustellen. Es ergab sich für 1\% ige Harnstofflösungen bei

Lange 1 ein Deficit von 22,2\% Stickstoff (im Mittel)

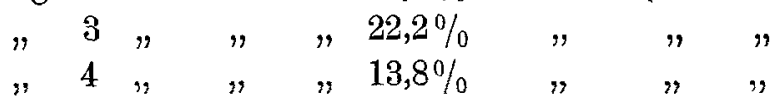

Diese verdünnte, aber in allen Lehrbüchern vorgeschriebene Lauge hat also einen sehr viel grösseren Correctionscoefficienten, als den Hüfner'schen.

Warum diese 3 nach Vorschrift hergestellten Laugen so verschieden wirkten, ergibt sich aus folgenden Thatsachen:

Lauge 1 war hergestellt aus $100 \mathrm{gr}$ Natron bydricum purissimum, i. e. alcohole depuratum und $25 \mathrm{~cm}$ gewöhnlichen käuflichen 
Ueber die Bestimmung des Harnstoffs im menschlichen Harne etc. 329

\begin{tabular}{|c|c|c|c|c|c|c|c|c|c|}
\hline 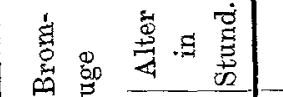 & $\stackrel{\infty}{-1}$ & $\stackrel{\infty}{\infty}$ & $\stackrel{\infty}{\infty}$ & $\stackrel{\infty}{=}$ & $\stackrel{\infty}{\infty}$ & $\stackrel{\infty}{-}$ & $\stackrel{\infty}{\infty}$ & $\stackrel{\infty}{\rightarrow}$ & $\stackrel{\infty}{\sim}$ \\
\hline$\dot{\theta}^{-i} \quad \dot{0}$ & $\pi$ & $H$ & $r-1$ & $\infty$ & $H$ & -1 & $H$ & $H$ & $H$ \\
\hline 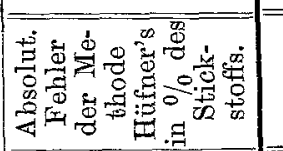 & $\sum_{\substack{\infty \\
\infty \\
\infty}}^{\infty}$ & $\stackrel{H}{+i}$ & $\stackrel{n}{\infty}$ & $\underbrace{+1}_{\substack{n \\
\infty}}$ & $\stackrel{i s}{i}$ & $\overrightarrow{\tilde{m}}$ & $\stackrel{0}{\infty}$ & $\overrightarrow{\mathfrak{N}}$ & $=$ \\
\hline 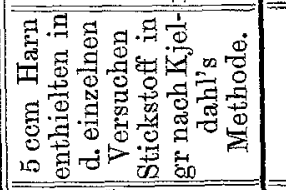 & $\begin{array}{l}0 \\
0 \\
0 \\
0 \\
0\end{array}$ & $\begin{array}{l}\infty \\
5 \\
6 \\
0 \\
0\end{array}$ & 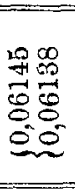 & $\approx$ & $=\underbrace{2}_{0}$ & 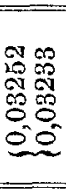 & & & 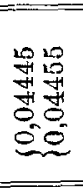 \\
\hline 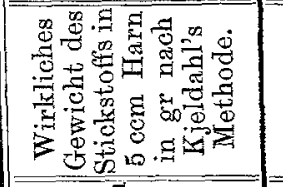 & $\begin{array}{l}0 \\
0 \\
0 \\
20 \\
0 \\
0\end{array}$ & $\begin{array}{l}0 \\
0 \\
0 \\
0 \\
0 \\
0\end{array}$ & $\begin{array}{l}\frac{1}{0} \\
0 \\
0\end{array}$ & $\begin{array}{l}\frac{D}{1} \\
\frac{0}{0} \\
0\end{array}$ & $\begin{array}{l}\frac{+1}{0} \\
0 \\
0\end{array}$ & $\begin{array}{l}\stackrel{+1}{0} \\
\text { @̊ } \\
0\end{array}$ & $\begin{array}{l}+ \\
0 \\
8 \\
0\end{array}$ & $\frac{10}{8}$ & $\frac{\mathfrak{s}}{5}$ \\
\hline 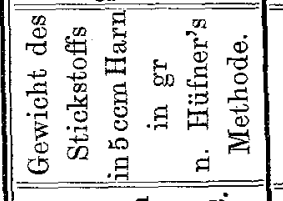 & $\begin{array}{l}\frac{0}{0} \\
0 \\
0 \\
0\end{array}$ & 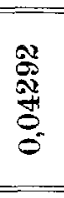 & $\begin{array}{l}0 \\
\frac{1}{10} \\
\frac{1}{0} \\
0 \\
0 \\
0\end{array}$ & $\begin{array}{l}9 \\
9 \\
\infty \\
0 \\
0 \\
0\end{array}$ & $\begin{array}{l}0 \\
\substack{8 \\
0 \\
0 \\
0}\end{array}$ & $\frac{\substack{0 \\
0}}{0}$ & 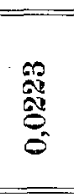 & 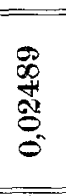 & 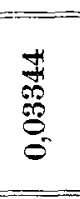 \\
\hline 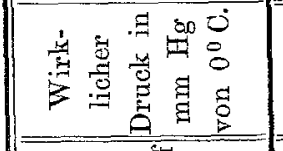 & 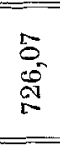 & 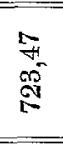 & $\begin{array}{l}\infty \\
\infty \\
\infty \\
1 \\
1\end{array}$ & $\frac{1}{i=}$ & 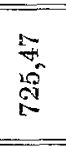 & $\begin{array}{l}\infty \\
\infty \\
0 \\
1 \\
1\end{array}$ & in & $\begin{array}{l}5 \\
0 \\
0 \\
0\end{array}$ & 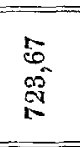 \\
\hline 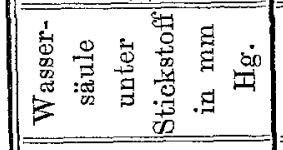 & $\stackrel{\infty}{0}$ & $\stackrel{10}{\text { is }}$ & 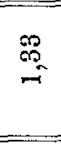 & $\stackrel{\infty}{\sigma o}$ & 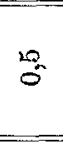 & 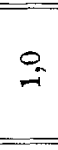 & $\stackrel{9}{\rightarrow}$ & $\sigma_{0}$ & की \\
\hline 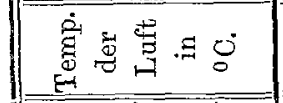 & 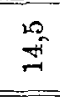 & $\stackrel{10}{t}$ & $\stackrel{H}{\stackrel{H}{-1}}$ & $\stackrel{20}{+1}$ & $\stackrel{10}{+4}$ & 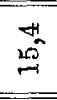 & \pm & $\underset{+1}{-10}$ & $\stackrel{10}{\sharp 1}$ \\
\hline 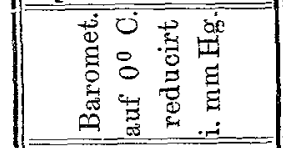 & $\frac{2}{8}$ & 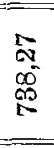 & $\vec{a}$ & $\underset{5}{5}$ & 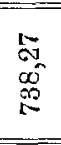 & $\frac{\vec{\sigma}}{a-1}$ & $\begin{array}{l}\widehat{\widehat{N}} \\
\infty 0 \\
\stackrel{0}{\infty}\end{array}$ & 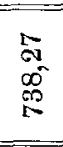 & $\begin{array}{l}\sqrt{\mathrm{N}} \\
\infty \\
\infty \\
\infty\end{array}$ \\
\hline 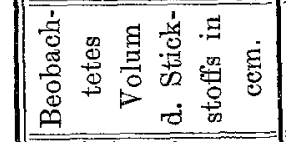 & $\stackrel{\oplus}{=}$ & $\begin{array}{c}a \\
\infty \\
-1\end{array}$ & in & $\stackrel{\vec{s}}{\rightarrow}$ & मु. & $\begin{array}{l}\infty \\
0 \\
-1\end{array}$ & 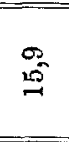 & 焉 & $\begin{array}{l}0 \\
0 \\
-\end{array}$ \\
\hline 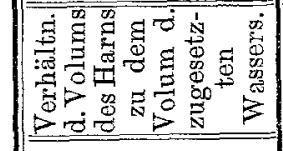 & $\begin{array}{l}\curvearrowright \\
\because \\
\sim\end{array}$ & $\begin{array}{l}o \\
\ddot{r}\end{array}$ & $\begin{array}{l}\mathbb{N} \\
\ddot{\sim}\end{array}$ & $\begin{array}{l}\sim \\
\ddot{\sim}\end{array}$ & $\begin{array}{l}\ddot{v} \\
\ddot{q}\end{array}$ & $\ddot{\sim}$ & $\ddot{\ddot{N}}$ & $\begin{array}{l}10 \\
\ddot{8}\end{array}$ & $\ddot{\ddot{z}}$ \\
\hline 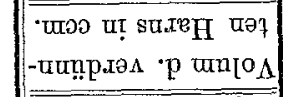 & to & $\begin{array}{l}\underset{*}{*} \\
20\end{array}$ & $\begin{array}{c}0 \\
0 \\
0 \\
10\end{array}$ & 8 & 8 & $\begin{array}{l}8 \\
8 \\
0\end{array}$ & $\begin{array}{l}80 \\
0 \\
0\end{array}$ & $\stackrel{8}{8}$ & 倠 \\
\hline 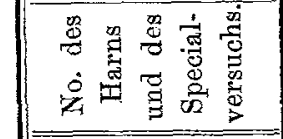 & $\tilde{r}$ & $\hat{N}$ & 鸹 & 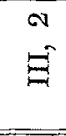 & $\hat{E}$ & $\vec{E}$ & 百 & $\vec{z}$ & $b^{-1}$ \\
\hline тәлnв $[7 . \bar{\mu}$ & - & $\infty$ & $\infty$ & $\nLeftarrow$ & 20 & $\infty$ & $N$ & $\infty$ & 0 \\
\hline
\end{tabular}


Broms. Letzteres war erst, nachdem die abgekühlte Natronlauge auf $1100 \mathrm{ccm}$ mit Wasser verdiunnt worden war, zugesetzt worden. Dann wurde das Volum auf $1250 \mathrm{cem}$ gebracht.

Lauge 3 ist aus denselben Ingredientien wie Lauge 1 dargestellt. Nur ist der abgekühlten Natronlange erst das Brom und dann das verdünnende Wasser zugefügt worden.

Lauge 4 ist aus $100 \mathrm{gr}$ Natron purissimum alcohole depuratum, $25 \mathrm{ccm}$ Bromum purissimum, welches langsam zur Vermeidung der Erhitzung der unverdünnten Lauge zugetröpfelt wurde, dargestellt. Diese Lauge wirkte nun auch viel energischer als die friiheren.

Es mögen nun in folgender Tabelle die Beobachtungsfehler aufgeführt werden, nachdem der jeder Lauge entsprechende Correctionscoefficient berijcksichtigt worden ist:

\section{Tabelle II.}

\begin{tabular}{|c|c|c|c|c|}
\hline $\begin{array}{c}\text { No. der Ver- } \\
\text { suche (S. die } \\
\text { Tabelle I). }\end{array}$ & $\begin{array}{l}\text { Absoluter } \\
\text { Fehler von } \\
\text { Hüfner's Me-- } \\
\text { thode in } \% \\
\text { d. Stickstoffs. }\end{array}$ & $\begin{array}{l}\text { Nummer } \\
\text { der } \\
\text { angewandt. } \\
\text { Laage. }\end{array}$ & $\begin{array}{l}\text { Zugehöriger } \\
\text { Beobach- } \\
\text { tungsfehler } \\
\text { der Brom- } \\
\text { lauge in } \\
\text { o\% des Stick- } \\
\text { stoffs. }\end{array}$ & $\begin{array}{l}\text { Corrigirter } \\
\text { Beobach- } \\
\text { tungsfehler } \\
\text { von Hüfner's } \\
\text { Methode i. } \% \\
\text { d. Stickstoffs. }\end{array}$ \\
\hline 1 & $-37,8$ & 1 & $+22,2$ & $-20,4$ \\
\hline 2 & $-24,4$ & 4 & $+13,8$ & $-12,3$ \\
\hline 3 & $-38,7$ & 1 & $+22,2$ & $-21,3$ \\
\hline $4:$ & $-37,4$ & 3 & $+22,2$ & $-19,6$ \\
\hline 5 & $-24,5$ & 4 & $+13,8$ & $-12,5$ \\
\hline 6 & $-39,1$ & 1 & $+22,2$ & $-21,9$ \\
\hline 7 & $-31,2$ & 4 & $+13,8$ & $-20,1$ \\
\hline 8 & $-29,1$ & 4 & $+13,8$ & $-17,7$ \\
\hline 9 & $-25,0$ & 4 & $+13,8$ & $-12,8$ \\
\hline
\end{tabular}

In der Arbeit Jacoby's, welche vor Kurzem aus Hiu fner's Laboratorium hervorgegangen ist, wird die Knop'sche Lauge unverdiunnt angewandt und es war also unsere Aufgabe, auch demgemäss zu analysiren. 
Ueber die Bestimmung des Harnstoffs im menschlichen Harne etc. 331

Diese concentrirten Laugen sind ron uns aus dem besten Material hergestellt, das Brom der Lauge erst zugesetzt, nachdem dieselbe absolut erkaltet war. Das Brom wurde nur tropfenweise zugefügt, während die Natronlauge in fast fortwährender Rotation sich befand und möglichst kühl gehalten wurde, dadurch, dass dio Flasche von kaltem Wasser umspült war.

Wir stellen zunächst die Versüche zusammen, welche wir mit 1\%iger Harnstofflösung anstellten, um den mittleren Correctionscoefficienten für unsere Laugen zu finden.

Siehe Tabelle III, p. 332.

Die Versuche ergeben also die Richtigkeit des Hüfner'sehen Correctionscoefficienten. Denn Hüfner's Zahl 4,2\% weicht von der unserigen $4,4 \%$ so wenig $a b$, als es unter diesen Verhältnissen nur erwartet werden kann.

Aus unserer Tabelle lassen sich noch einige Folgerungen $a b-$ leiten. Hüfner benutzt nur Laugen, die über Nacht gestanden. Alle mit $a$ bezeichneten Laugen sind 6 , die mit $b$ bezeichneten 20 Stunden alt.

Tabelle IV.

\begin{tabular}{|c|c|c|c|c|c|}
\hline $\begin{array}{c}\text { No. der } \\
\text { Versuche } \\
\text { (S. Tab. III). }\end{array}$ & $\begin{array}{l}\text { No. der } \\
\text { Lange. }\end{array}$ & $\begin{array}{c}\text { Alter der } \\
\text { Lauge } \\
\text { in Stunden. }\end{array}$ & \begin{tabular}{|c||} 
Absoluter \\
Fehler der \\
Methode i. $\%$ \\
d.Stickstoffs.
\end{tabular} & $\begin{array}{l}\text { Alter der } \\
\text { Lauge } \\
\text { in Stunden. }\end{array}$ & $\begin{array}{l}\text { Absoluter } \\
\text { Fehler der } \\
\text { Methode i. } \% \\
\text { d. Stickstoffs. }\end{array}$ \\
\hline 10 & II a & 6 & $-0,37$ & & \\
\hline 11 & II a & 6 & $-4, \mathbf{1}$ & & \\
\hline 12 & II $\mathbf{b}$ & & & 20 & $-5,0$ \\
\hline 13 & II $b$ & & & 20 & $-2,4$ \\
\hline 16 & IV a & 6 & $-5,0$ & & \\
\hline 17 & $\mathrm{IV} \mathrm{a}$ & 6 & $-5,9$ & & \\
\hline 18 & IV a & 6 & $-6,3$ & & \\
\hline 19 & IVb & & & 20 & $-6,7$ \\
\hline 20 & IV b & & & 20 & $-5,0$ \\
\hline 21 & IV b & & & 20 & $-6,7$ \\
\hline Mittel: & & & $-4,3$ & & $-5,1$ \\
\hline
\end{tabular}




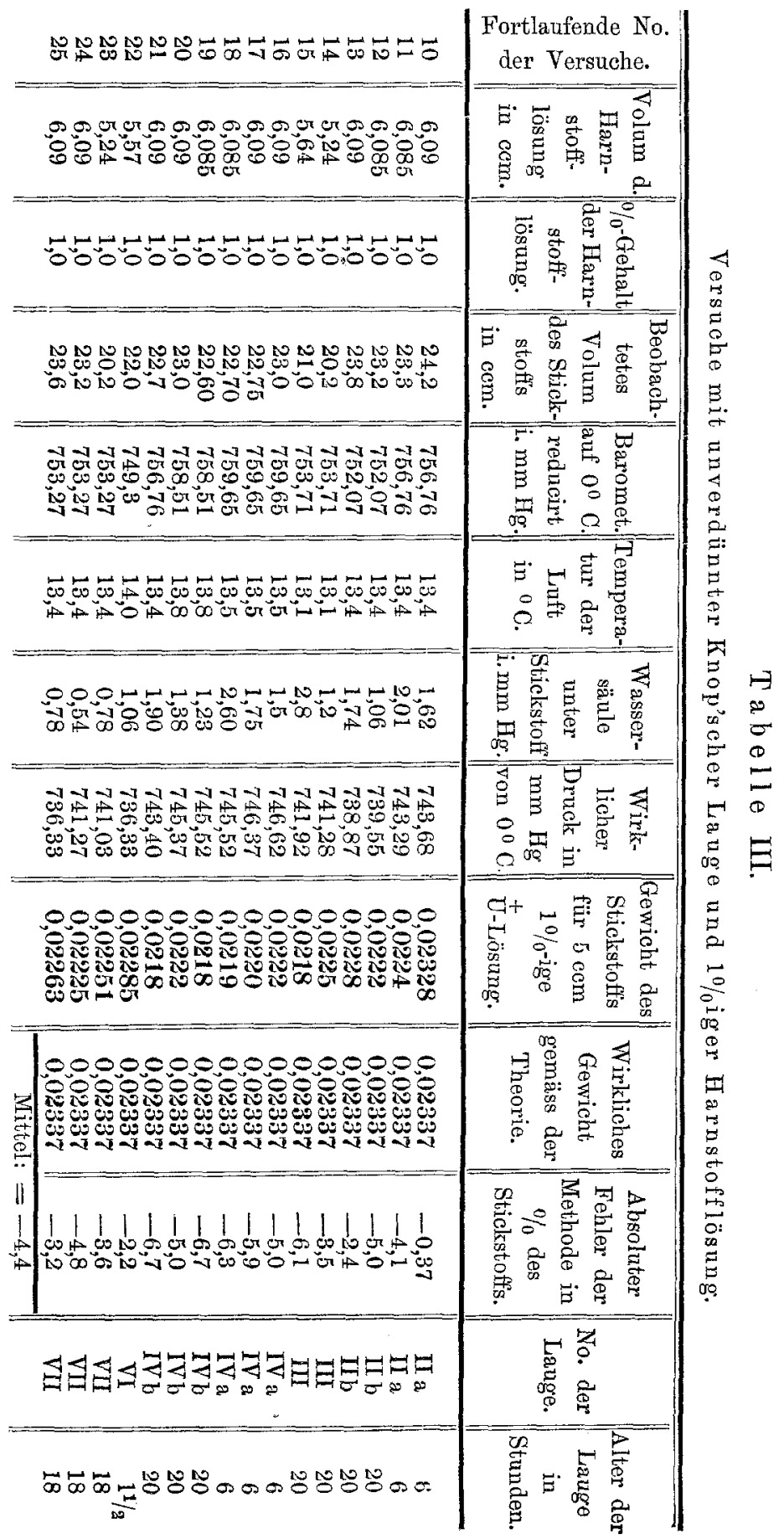


Ueber die Bestimmung des Harnstoffs im menschlichen Harne etc. 333

Die Tabelle IV lehrt, dass die frische Lauge von 6 Stunden Alter etwas kräftiger als solche $\mathrm{zu}$, wirken scheint, die bereits 20 Stunden gestanden hat.

Wie schon Hüfner bemerkt hat, geben im Allgemeinen seine Apparate ein grösseres Deficit an Stickstoff, wenn die die Harnstofflösung aufnehmende Kapsel ein grösseres Volum hat, weshalb er ein solches von circa $5 \mathrm{ccm}$ vorschreibt.

Stellen wir zur Prüfung dieser Frage alle vergleichbaren Versuche zusammen in Tabelle V:

Tabelle V.

Alle Harnstofflösungen sind $1 \%$-ige.

\begin{tabular}{|c|c|c|c|c|c|}
\hline $\begin{array}{c}\text { No. der } \\
\text { Versuche } \\
\text { (S. Tab. III). }\end{array}$ & $\begin{array}{l}\text { No. der } \\
\text { Lauge. }\end{array}$ & $\begin{array}{l}\text { Volum der } \\
\text { Harnstoff- } \\
\text { lösung } \\
\text { in ecm. }\end{array}$ & $\begin{array}{c}\text { Beobach- } \\
\text { tungsfehler } \\
\text { in } \% \text { des } \\
\text { Stickstoffs. }\end{array}$ & $\begin{array}{l}\text { Volum der } \\
\text { Harnstoff- } \\
\text { lösung in } \\
\text { ccm. }\end{array}$ & $\begin{array}{l}\text { Beobach- } \\
\text { tungsfehler } \\
\text { in } \% \text { des } \\
\text { Stickstoffs. }\end{array}$ \\
\hline 14 & III & 5,24 & $-3,5$ & & \\
\hline 15 & III & & & 5,64 & $-6,1$ \\
\hline 18 & IV a & 6,085 & $-6,3$ & & \\
\hline 16 & $I \nabla a$ & & & 6,09 & $-5,0$ \\
\hline 17 & IV a & & & 6,09 & $-5,9$ \\
\hline 19 & $\mathrm{IVb}$ & 6,085 & $-6,7$ & & \\
\hline 20 & IVb & & & 6,09 & $-5,0$ \\
\hline 21 & $\mathrm{IVb}$ & & & 6,09 & $-6,7$ \\
\hline 23 & VII & 5,24 & $-3,6$ & & \\
\hline 24 & VII & & & 6,09 & $-4,8$ \\
\hline 25 & VII & & & 6,09 & $-3,2$ \\
\hline Mittel: & & 5,65 & $-5,0$ & 6,08 & $-5,2$ \\
\hline
\end{tabular}

Wir gehen nun über zur Mittheilung der Versuche, welche mit Harn angestellt wurden und übersichtlich in Tabelle VI aufgeführt stehen. 


\begin{tabular}{|c|c|c|c|c|c|c|c|c|c|c|c|c|}
\hline 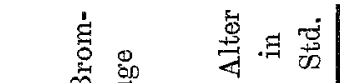 & 0 & 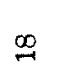 & $\varphi$ & 오 & 0 & $\stackrel{\infty}{\infty}$ & 0 & ㅇ & $\theta$ & $\stackrel{\infty}{\sim}$ & శి & 200 \\
\hline 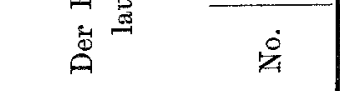 & $\stackrel{\pi}{\pi}$ & in & $\stackrel{2}{E}$ & $\stackrel{e}{E}$ & $\stackrel{\infty}{-}$ & $\frac{2}{m}$ & $\mathscr{E}$ & $\begin{array}{l}0 \\
\theta\end{array}$ & $\infty$ & R & $=$ & $\Xi$ \\
\hline 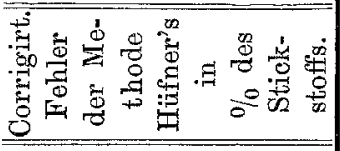 & $\sigma_{1}^{10}$ & $\begin{array}{l}10 \\
6 \\
1\end{array}$ & $\stackrel{a}{1}$ & $\begin{array}{l}\infty \\
0 \\
0 \\
1\end{array}$ & $\stackrel{\infty}{\infty}$ & $\overrightarrow{0}$ & 1 & in & $\stackrel{\infty}{\stackrel{\infty}{Q}}$ & $\begin{array}{c}\infty \\
\infty \\
1\end{array}$ & $\stackrel{\infty}{1}$ & $\stackrel{1}{10}$ \\
\hline 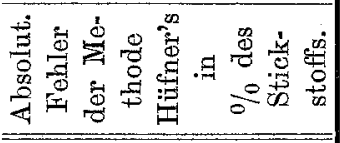 & $\overbrace{1}^{\infty 00}$ & $\stackrel{0}{0}$ & $\stackrel{0}{a}$ & $\stackrel{H}{1}$ & $\stackrel{\infty}{\infty}$ & $\stackrel{\infty}{0}$ & $\begin{array}{c}0 \\
\infty \\
1\end{array}$ & $\begin{array}{c}20 \\
100 \\
1\end{array}$ & $\prod_{1}^{\infty}$ & $\stackrel{\infty}{\mathfrak{A}}$ & $\stackrel{10}{1}$ & $\stackrel{H}{=}$ \\
\hline 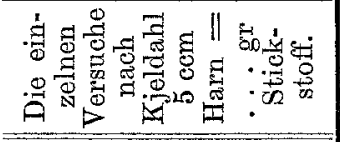 & & $\begin{array}{r}20 \\
10 \\
0 \\
0 \\
\end{array}$ & $\begin{array}{l}\infty \\
\frac{1}{9} \\
0 \\
0 \\
0\end{array}$ & & & 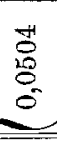 & $\begin{array}{l}0 \\
\stackrel{0}{0} \\
0 \\
0\end{array}$ & & & $\begin{array}{l}5 \\
\dot{H} \\
0\end{array}$ & $\begin{array}{l}0 \\
\stackrel{0}{H} \\
0 \\
0\end{array}$ & \\
\hline 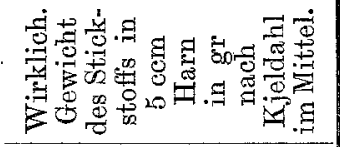 & & $\frac{2}{2}$ & & & & 赵 & & . & & 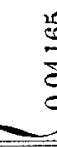 & & \\
\hline 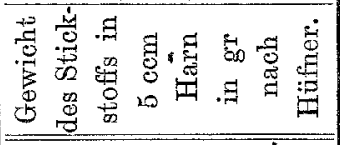 & $\begin{array}{l}10 \\
8 \\
8 \\
0 \\
0\end{array}$ & $\begin{array}{l}0 \\
\stackrel{8}{1} \\
0 \\
0\end{array}$ & 蛢 & $\begin{array}{l}8 \\
\stackrel{8}{0} \\
8\end{array}$ & 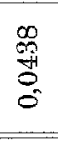 & $\begin{array}{l}\frac{1}{0} \\
0 \\
0\end{array}$ & $\begin{array}{l}8 \\
\stackrel{8}{0} \\
0\end{array}$ & $\stackrel{5}{E}$ & $\begin{array}{l}10 \\
20 \\
0 \\
0 \\
0\end{array}$ & $\begin{array}{l}\infty \\
0 \\
0 \\
0 \\
0 \\
0\end{array}$ & $\begin{array}{l}\infty \\
\stackrel{\infty}{8} \\
0 \\
0 \\
0\end{array}$ & $\begin{array}{l}0 \\
0 \\
\infty \\
0 \\
0\end{array}$ \\
\hline 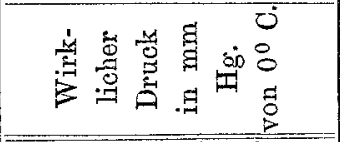 & 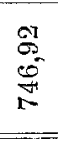 & 昰 & $\vec{E}$ & $\begin{array}{l}8 \\
\infty \\
\infty \\
0 \\
0\end{array}$ & 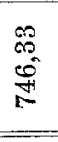 & 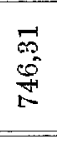 & $\begin{array}{l}\stackrel{8}{8} \\
\text { on } \\
\text { It }\end{array}$ & 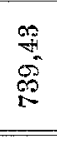 & 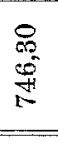 & 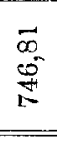 & $\underbrace{\infty}_{i}$ & 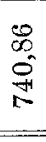 \\
\hline 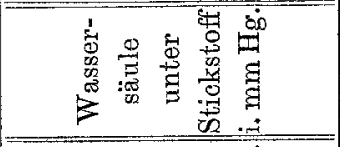 & $\underset{-}{g}$ & $\stackrel{8}{8}$ & $\stackrel{8}{8}$ & $\begin{array}{l}\text { चे } \\
\text { ôे }\end{array}$ & $\stackrel{\infty}{\stackrel{\infty}{=}} \stackrel{-}{=}$ & $\mathscr{S}_{-1}$ & $\begin{array}{l}H \\
\sigma_{0}^{H} \\
0\end{array}$ & $\stackrel{\uplus}{\leftrightarrows}$ & $E$ & $\stackrel{0}{4}$ & $\frac{0}{0}$ & 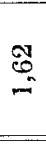 \\
\hline 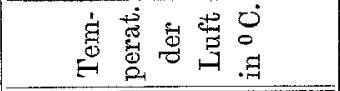 & $\stackrel{20}{\cos }$ & $\underset{+i}{g}$ & की & $\underset{\infty}{H}$ & $\stackrel{10}{10}$ & $\stackrel{\circ}{ \pm}$ & $\stackrel{+}{-1}$ & $\stackrel{+}{=}$ & $\stackrel{10}{10}$ & $\stackrel{0}{-1}$ & $\stackrel{4}{+1}$ & $\overrightarrow{05}$ \\
\hline 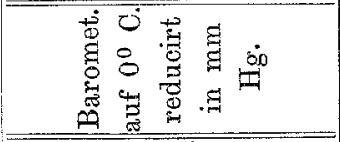 & 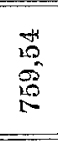 & $\stackrel{\infty}{8}$ & $\begin{array}{l}6 \\
6 \\
60 \\
10 \\
10\end{array}$ & के & $\begin{array}{l}20 \\
20 \\
10 \\
10\end{array}$ & $\frac{\infty}{8}$ & $\begin{array}{l}0 \\
10 \\
18 \\
10\end{array}$ & 总 & 紫 & $\frac{\infty}{8}$ & $\begin{array}{l}5 \\
\text { of } \\
\text { 总 }\end{array}$ & $\begin{array}{l}E \\
80 \\
50\end{array}$ \\
\hline 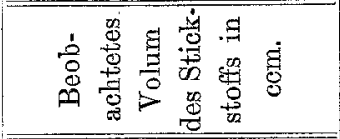 & $\stackrel{0}{=}$ & $\underset{\infty}{\infty}$ & $\stackrel{-1}{\infty}$ & 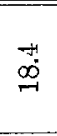 & $\stackrel{0}{ \pm}$ & $\stackrel{2}{\pi}$ & $\underset{-}{+}$ & $\underbrace{\infty}_{-10}$ & $\begin{array}{l}H^{\prime} \\
\stackrel{\infty}{-1}\end{array}$ & $\stackrel{\infty}{\infty}$ & $\begin{array}{l}\infty \\
= \\
=\end{array}$ & $\begin{array}{l}\sigma \\
\sigma^{2}\end{array}$ \\
\hline 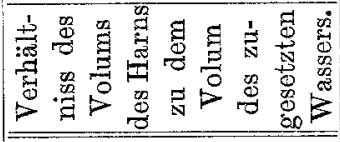 & $\ddot{r}$ & $\overrightarrow{-}$ & $\begin{array}{l}\ddot{r} \\
\ddot{r}\end{array}$ & $\stackrel{-1}{\ddot{\sim}}$ & $\stackrel{o}{\stackrel{\alpha}{-1}}$ & $\stackrel{\sim}{\dddot{\sim}}$ & $\stackrel{N}{\ddot{\sim}}$ & $\begin{array}{l}a \\
\ddot{-}\end{array}$ & $\overrightarrow{\ddot{r}}$ & $\vec{\sim}$ & $\ddot{\ddot{n}}$ & $\ddot{\ddot{Z}}$ \\
\hline 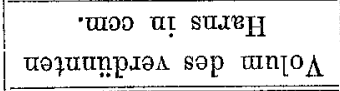 & $\begin{array}{l}\text { तn } \\
10\end{array}$ & $\begin{array}{l}\text { त } \\
\text { in }\end{array}$ & $\begin{array}{l}\text { त् } \\
\text { is }\end{array}$ & $\begin{array}{l}\text { N } \\
\text { in }\end{array}$ & $\begin{array}{l}\text { से } \\
100\end{array}$ & $\begin{array}{l}0 \\
0 \\
0\end{array}$ & $\begin{array}{l}15 \\
100 \\
10\end{array}$ & $\begin{array}{l}50 \\
10\end{array}$ & 8 & 8 & $\begin{array}{l}\text { E0 } \\
15\end{array}$ & 8 \\
\hline 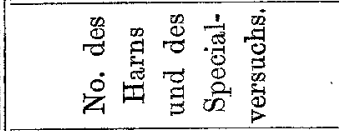 & $\vec{r}$ & $\stackrel{\infty}{\infty}$ & $\stackrel{-1}{-1}$ & H & $\vec{E}$ & $\stackrel{\infty}{\nexists}$ & 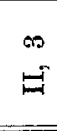 & E & $\vec{\Xi}$ & $\hat{E}$ & $\Xi$ & キ \\
\hline $\mathrm{o}_{\mathrm{N}}$ & $\overline{\mathscr{\sigma}}$ & $\stackrel{5}{\circ}$ & 总 & $\bar{A}$ & 8 & $\vec{\infty}$ & $\widehat{c o}$ & $\infty$ & का & 80 & Co & $\bar{a}$ \\
\hline
\end{tabular}


Ueber die Bestimmung des Harnstoffs im menschlichen Harne etc. 335

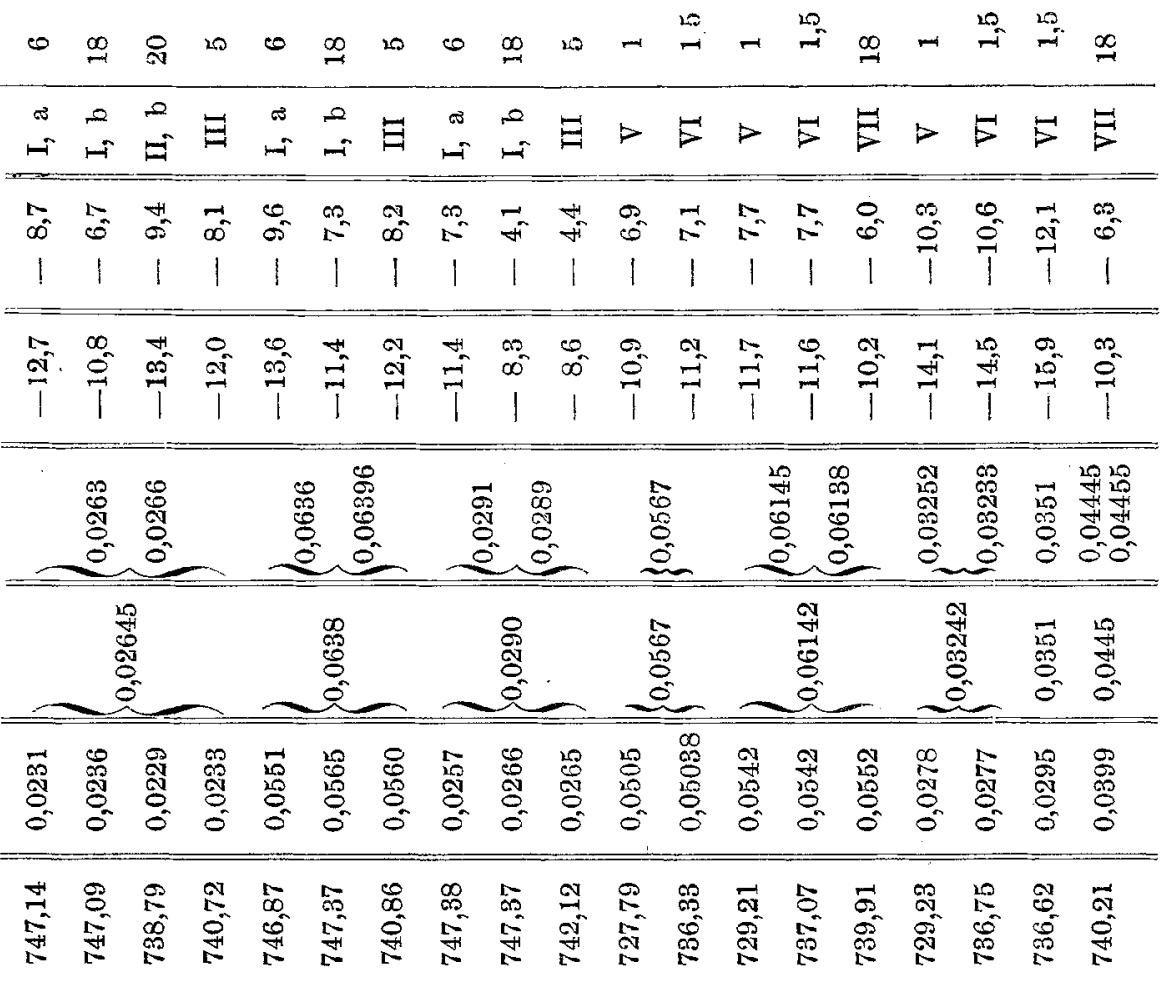

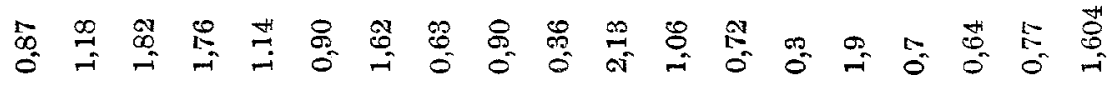

की भी मी की की

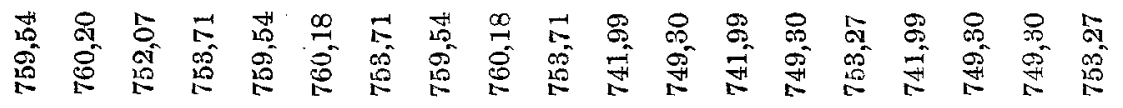

H.

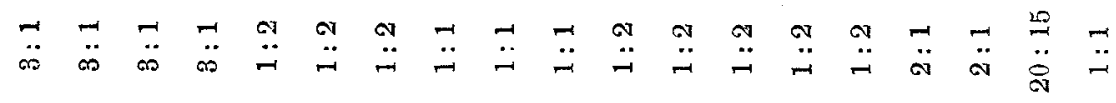

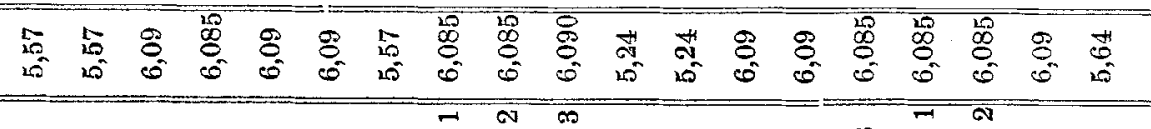

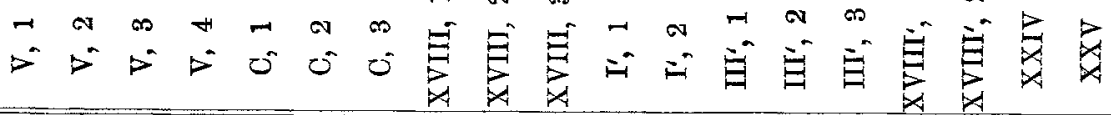

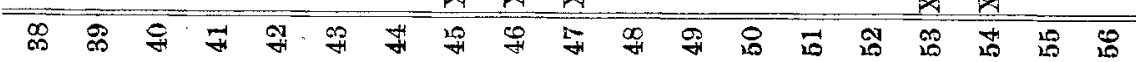


336 E. Pflüger u. Friedrich Schenck: Ueber die Bestimmung etc.

Die mitgetheilte Untersuchung lehrt, was iibrigens Jacoby in oben citirter Arbeit jetzt auch anerkennen musste, dass die noch in neuesten Lebrbiichern vertretene Ansicht, als liefere die Knop-Hüfner'sche Analyse sehr nahe den Gesammtstickstoff des Harns, nicht richtig ist. Sie giebt den letzteren Werth trotz der Hüfner'schen Addition und trotz des aus Ammoniaksalzen, Harnsäure, Kreatinin u. s. w. noch hinzukommenden Stickstoffs um $7,5 \%$ im Mittel zu klein. Das Deficit schwankte von 1,2 bis $12,1 \%$.

Wenn man die bisher stillschweigend allgemein gemachte Annahme zulässt, dass es im Harne keinen Körper gibt, der den Hüfn er'schen Corrections-Coefficienten vergrössert, so zeigt unsere Untersuchung, dass neben dem Harnstoff im Harne eine viel grössere Menge stickstoffhaltiger Körper vorkommt, als man dies nach den gegenwärtigen Kenntnissen zu erwarten berechtigt war.

Diese Annahme ist aber nicht mehr zulässig. Aus unseren mit verdünnter Lauge ausgeführten Versuchen (siehe Tabelle I u. II) ergibt sich, dass trotz entsprechender im Sinne Hüfner's ausgeführter Correctur das Verhältniss des Harnstoffs zu den übrigen stickstoffhaltigen Körpern ein viel kleineres ist, als in den mit unverdünnter K nop'scher Lauge angestellten Analysen ermittelt wurde. Die in gebräuchlicher Weise verdiunnte Lauge gibt also trotz Correctur einen falschen und zwar viel zu niedrigen Werth für den Harnstoff. Dies ist kaum anders zu deuten als dureh die Annahme, dass die verdünnte Lauge aus einer Lösung chemisch reinen Harnstoffs viel mehr Stickstoff frei macht als ans einer solchen, die wie der Harn neben dem Harnstoff noch andere Körper enthält. Die Reaction hat dann theilweise einen anderen Verlauf. Wenn nun der Einfluss dieser den Hï fner'sehen Corrections-Coefficienten vergrössernden Körper bei Anwendung unverdünnter Kn op'scher Lauge auch geschwächt wird, so ist es doch nicht wabrscheinlich, dass er ganz beseitigt werde.

Da man bei der wechselnden Zusammensetzung des Harnes niemals weiss, um wie viel, ja eigentlich nicht einmal in welchem Sinne der Hüfner'sche Coefficient geändert ist, so verliert die Anwendung desselben ibre Berechtigung und die Methode ihre Basis, bis der Beweis erbracht ist, dass der für den Harnstoff als solchen nach Hüfner ermittelte Werth mit einem nur irrelevanten Fehler behaftet ist. Diese Aufgabe ist deshalb nicht so leicht zu entscheiden, weil wir bis jetzt keine controlirte Methode haben, um den Harnstoff im Harne einigermaassen genau zu bestimmen. 